\title{
Prognosis of hospitalized children under 2 years of age with co-detection of influenza $A$ and respiratory syncytial virus at the healthcare facility
}

Elisa Teixeira Mendes 1

iD https://orcid.org/0000-0003-4251-8185

Hadassa L. Paranhos 2

iD https://orcid.org/0000-0001-7588-8794

Isabela C. M. Santos 3

iD https://orcid.org/0000-0003-1319-5620

Lais Bomediano de Souza 4

iD https://orcid.org/0000-0002-8980-0690

\author{
José Luis Braga de Aquino 5 \\ (iD https://orcid.org/0000-0002-0604-9054 \\ Vania Aparecida Leandro-Merhi 6 \\ iD https://orcid.org/0000-0002-2623-6471 \\ Raquel Vieira da Silva 7 \\ iD https://orcid.org/0000-0002-2825-880X \\ Maria Patelli J. S. Lima 8 \\ (iD https://orcid.org/0000-0002-6066-375X
}

\footnotetext{
1-8 Programa de Pós-Graduação em Ciências da Saúde. Centro de Ciências da Vida. Faculdade de Medicina. Pontifícia Universidade Católica de Campinas. Av John Boyd Dunlop, s.n. km 6,9. Jardim Londres. Campinas, SP, Brasil. CEP: 13.034-685. E-mail: elisatmendes@gmail.com
}

\begin{abstract}
Objectives: the aim of this study is to evaluate the impact of co-detection of Flu A and $R S V$ using rapid immunochromatographic tests at the point of care, in pediatric patients under 2 years of age in a general hospital.

Methods: a retrospective cohort study was conducted to analyze clinical outcomes in hospitalized infants with viral respiratory disease with positive results of rapid immunochromatographic test for RSV and/or Flu-A, from 2013 to 2018. A logistic regression model was adjusted to analyze predictors of orotracheal intubation during hospitalization.

Results: we analyzed 220 cases: RSV (192), Flu-A (9), co-detection (19). Lethality rate was $1.8 \%$ (2 cases), and $88 \%$ (194) were under 1 year of age. Mean time of hospitalizations was higher in patients with co-detection. Variables significantly associated with orotracheal intubation were: younger age in months, comorbidities, RSV and Flu-A co-detection, and bacterial pneumonia during hospitalization.

Conclusions: RSV and Flu-Aco-detection was associated with the least favorable clinical prognoses in this study. Rapid test diagnosis may provide important information at the point of care, because molecular panels are not widely accessible in general hospitals. Rapid diagnosis allows timely evaluation and treatment.
\end{abstract}

Key words Respiratory syncytial virus, Influenza virus, Point of care testing, Prognosis

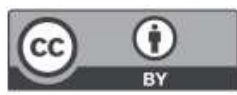




\section{Introduction}

Respiratory syncytial virus (RSV) and influenza (Flu) virus contribute substantially to the overall burden of severe respiratory tract infection in children. Of all respiratory viruses, RSV is estimated to be the main cause of hospitalizations for children under 2 years of age worldwide, and the first year of life is the period with the highest risk of infection.1-3 Both RSV and Flu viruses have seasonal incidence patterns, although there are annual variations in the number of cases and in fatality rates. 4,5 The burden of morbidity and mortality caused by these respiratory viral infections in children has been reported in several studies in Brazil and other parts of the world. $1,5,6$

A rapid diagnosis is necessary to determine antiviral therapy early (especially for Influenza) and to apply hospital infection control measures.7 Molecular information from viral panels using reverse transcriptase polymerase chain reaction (RTPCR) is sensitive and facilitates accurate diagnosis, but this technology is expensive and not available in most health services, especially public hospitals. ${ }^{8}$ In addition, with the exception of rapid molecular assays tests, the results can take a few days, especially if the sample is sent to public health reference laboratories, delaying the treatment decision and implementation. In contrast, immunochromatographic tests, such as a rapid influenza diagnostic test (RIDT), although less sensitive, can be performed at bedside and provide valuable information in a few minutes. ${ }^{9}$ Rapid tests are also important to reduce antibiotic use, and can identify viral coinfection, ${ }^{9}$ particularly relevant in the context of the SARS CoV-2 epidemic.

Viral co-detection has been reported in the literature frequently; however, the clinical and prognostic impact, particularly in children, is still controversial.10-13 It is more common in children under one year of age, probably due to delay in the development of upper respiratory viral clearance capacity and an immature immune system, but it is unclear whether such cases involve co-infection or only co-detection.?

The aim of this study is to evaluate the association between co-detection of FluA virus and RSV on the prognosis and clinical outcomes of children diagnosed by rapid tests performed at the point of care.

\section{Methods}

This is a retrospective cohort study to evaluate clinical outcomes in children $<2$ years old, hospitalized from 2013 to 2018 with respiratory viral infection with a positive rapid test for FluA, RSV or both in the pediatric department of the Pontifical Catholic University of Campinas Hospital (PUC-Campinas Portuguese) in the city of Campinas (approximately 1.2 million inhabitants), São Paulo State. It is a 325bed university hospital with an 8-bed pediatric ICU and 16-bed neonatal intensive care unit. The hospital receives referrals from primary care and other health services in the municipality and the metropolitan region, especially from low-income areas.

Severe acute respiratory syndrome (SARS) is defined as any patient with symptoms of fever, even if referred, accompanied by cough or sore throat and with dyspnea or $\mathrm{O}_{2}$ saturation $<95 \%$ or respiratory distress. Observing the biosecurity measures, every child with SARS and hospitalization criteria, underwent RSV and Flu rapid immunochromatographic test (RICT) for qualitative detection of RSV fusion protein, and Flu A/B antigen. One sample of nasal secretion aspirate was collected using a disposable plastic collector bottle with vacuum control for each test, according to Ministry of Health protocols of influenza surveillance. RICT was performed just after sample collection. RICT use is routinely performed in our service, and this practice brings security to medical assistance in the point of care, facing the high cost of molecular testing, and lack of availability in our public health service.

We used two rapid tests (RT): an in vitro immunochromatographic assay for detection Flu virus and an assay for the detection of RSV fusion protein antigen, both using Alere ${ }^{\circledR}$ technology, United Kingdom, Scotland.

We analyzed retrospectively all medical records of patients under 2 years of age with a respiratory condition requiring hospitalization and who underwent rapid test from a sample of nasopharyngeal aspirate or swab with positive results for RSV and/or Flu-A. Patients with negative results for both tests were excluded. This age group was chosen because it represents the majority of severe cases of viral respiratory conditions. 3,12

The following study variables were recorded: age in months, length of hospital stay, signs and symptoms at admission, comorbidities, prematurity, diagnosis of bacterial pneumonia during hospitalization, orotracheal intubation (occurrence and time of intubation), positive rapid test for RSV only, FluA only, or both, ICU admission, and clinical outcome (death or discharge). The diagnosis of bacterial pneumonia was defined in children with SARS, radiological infiltration, and introduction of antimicrobials by the pediatrician. 
Temporal distribution of cases according to dates and length (in days) of hospitalization were analyzed. The proportions of categorical variables were compared using Pearson's chi-square test, and variables with continuous data were analyzed via ANOVA or Kruskal-Wallis test when variance was not homogeneous. Orotracheal intubation (OTI) was considered a categorical parameter of infection severity (yes/no). To access association between the study variables and the outcome (OTI) an initial logistic regression model included all the variables that presented an association at a level of $p<0.20$ in the univariate analysis. Then, a multiple logistic regression model was adjusted and those variables that presented a $p$ value lower than 0.05 remained in the final model.

We used Excel 2013 for database construction and time series graph; SPSS v. 21 was used for the statistical analysis.

The study was approved by the Research Ethics Committee, Pontifícia Universidade Católica de Campinas (recommendation process number: 2.727.512)

\section{Results}

The lethality rate of the study population $(n=220)$ was $1.8 \%(n=4)$ : two premature children under 6 months, one child had fatal Flu A infection complicated by chronic neuropathy with $\mathrm{O}_{2}$ dependence who underwent gastrostomy and tracheostomy, and the last child without comorbidities with bronchiolitis associated bacterial lung infection. Two children were not included in the analysis due to transfer to other services.

Table 1 shows the demographic, epidemiological, and clinical variables according to positive RT results for RSV-only, FluA only, and RSV+FluA, comparing the proportion between groups of test results. No children tested positive for FluB. RT identified 192 (87.3\%) RSV-only infections and 19 (8.6\%) co-detections.

Although we include children $<2$ years old, 194 $(88.2 \%)$ were younger than 1 year and $160(72.7 \%)$ were younger than 6 months. Twenty-nine (13.2\%) patients were premature, and of these, only 3 $(10.3 \%)$ were classified as "very preterm" according to the World Health Organization (28-30 weeks of pregnancy); there were no cases of extreme prematurity. Only 29 (13.2\%) patients had no associated clinical conditions (congenital heart disease, Down syndrome, congenital gastrointestinal malformations, renal failure, or bronchopulmonary dysplasia). Length of hospital stay was longer and OTI was more frequent among those with viral co-detection at the time of admission. We observed a lower frequency of intubated children among those with RSV infection only.

Fever was more common among those with positive rapid tests for Flu-A and co-infection with both viruses (FluA and RSV) compared with RSV only. Children with RSV+FluA co-detection had a longer hospitalization (Figure 1) and more frequently underwent orotracheal intubation, the intubation time was similar among children who underwent this procedure (Table 1).

Variables associated with OTI, as a measure of severity, and poor clinical outcome were: younger age in months, comorbidities, viral co-detection (FluA and RSV), and diagnosis of bacterial pneumonia during hospitalization (Table 2). Detection of isolated Flu-A was not associated with OTI in the univariate or multivariate analysis. Of the 33 cases of pneumonia, we found 9 isolated microorganisms in 8 patients: Moraxella catarrhalis, Haemophilus influenza, Enterobacter cloacae, Klebsiella pneumoniae, Pseudomonas aeruginosa, Staphylococcus aureus and Streptococcus pneumoniae.

We also observed a clear seasonality of hospitalizations with marked peaks in April through June, which are months of greater circulation of the RSV and Flu viruses, respectively (Figure 2).

\section{Discussion}

In this study, we evaluated the records of 220 patients with confirmed FluA and/or RSV who were $<2$ years of age on admission, during a 6-year period. RSV had a higher incidence in this study $(87.3 \%)$ than Flu viruses (4.1\%). $3-5,14$ Higher incidence of RSV occurs even at peak periods of Flu virus circulation, 11 especially in children $<2$ years of age who are more frequently hospitalized for respiratory conditions. 3,12

The most severe symptoms, signaled by indication for OTI, were detected in younger children, with comorbidities, with a diagnosis of bacterial pneumonia and those with viral co-detection. The role of comorbidities and bacterial pneumonia aggravating viral respiratory conditions is well described in the literature.1,14-17 Particularly influenza infection has been associated with secondary bacterial infection in up to $65 \%$ of cases in a previous meta-analysis. 16

Although the number of cases of influenza was small in our point-of-care study, co-detection was associated with the poorest prognosis, suggested by a higher risk of OTI, ICU admission, and longer hospital stay. Studies evaluating viral co-detection 
Table 1

Clinical variables associated with RSV and influenza detection in children $<2$ years hospitalized at PUC-Campinas Hospital, 2013 to 2018.

\begin{tabular}{|c|c|c|c|c|c|c|c|c|c|}
\hline \multirow[t]{2}{*}{ Variables } & \multicolumn{2}{|c|}{ RSV } & \multicolumn{2}{|c|}{ FluA } & \multicolumn{2}{|c|}{ RSV+FluA } & \multicolumn{2}{|c|}{ Total } & \multirow[t]{2}{*}{$p$} \\
\hline & $\mathrm{n}$ & $\%$ & $\mathrm{n}$ & $\%$ & $\mathrm{n}$ & $\%$ & $\mathrm{n}$ & $\%$ & \\
\hline Age & & & & & & & & & 0.01 \\
\hline$<6$ months & 145 & 75.5 & 2 & 22.2 & 13 & 68.4 & 160 & 72.7 & \\
\hline 6 to 1 year & 26 & 13.5 & 4 & 44.4 & 4 & 21.1 & 34 & 15.5 & \\
\hline 1 to 2 years & 21 & 11.4 & 3 & 33.3 & 2 & 10.5 & 26 & 11.8 & \\
\hline Prematurity & 21 & 23.1 & 3 & 33.3 & 5 & 26.3 & 29 & 13.2 & \\
\hline Comorbidities** & 24 & 12.7 & 0 & - & 5 & 26.3 & 29 & 13.2 & 0.05 \\
\hline Bacterial Pneumonia & 24 & 12.5 & 2 & 22.2 & 7 & 36.8 & 33 & 15.0 & 0.15 \\
\hline \multicolumn{10}{|l|}{ Symptoms / Signs } \\
\hline Fever & 127 & 66.2 & 9 & 100.0 & 17 & 89.5 & 153 & 68.6 & 0.01 \\
\hline Cough & 177 & 84.3 & 8 & 88.9 & 17 & 89.5 & 202 & 91.8 & 0.91 \\
\hline Wheezing & 185 & 88.1 & 3 & 33.3 & 16 & 84.2 & 189 & 85.9 & $<0.01$ \\
\hline Cyanosis & 36 & 18.8 & 0 & - & 7 & 36.8 & 43 & 19.3 & 0.03 \\
\hline Whoop.Cough Synd. & 6 & 3.1 & 1 & 11.1 & 0 & - & 7 & 3.1 & 0.29 \\
\hline \multicolumn{10}{|l|}{ Outcome } \\
\hline Hospital (days) $\overline{\mathrm{X}}(\mathrm{SD})$ & \multicolumn{2}{|c|}{$9.2(9.4)$} & \multicolumn{2}{|c|}{$10.2(6.6)$} & \multicolumn{2}{|c|}{$17.9(11.9)$} & \multicolumn{2}{|c|}{$10.0(9.8)$} & $<0.01+$ \\
\hline Oxygen therapy & 191 & 99.5 & 3 & 33.3 & 19 & 100.0 & 214 & 96.8 & $<0.01$ \\
\hline $\mathrm{ICU}^{* * *}$ & 57 & 29.7 & 2 & 22.2 & 15 & 79.0 & 74 & 33.6 & $<0.01$ \\
\hline $\mathrm{OTI} * * * *$ & 50 & 26.0 & 6 & 66.7 & 14 & 73.7 & 70 & 31.8 & $<0.01$ \\
\hline OTI (days) $\bar{x}(S D)$ & \multicolumn{2}{|c|}{$2.4(9.2)$} & \multicolumn{2}{|c|}{$8,7(5.2)$} & \multicolumn{2}{|c|}{$13.1(5.2)$} & \multicolumn{2}{|c|}{$3.9(7.4)$} & $0.16 \neq$ \\
\hline Total & 192 & 87.3 & 9 & 4.1 & 19 & 8.6 & 220 & 100.0 & \\
\hline
\end{tabular}

* Pearson chi-square test; **Comorbidity= congenital heart disease; Down syndrome, other GIT congenital malformation, renal failure, bronchopulmonary dysplasia; RSV $=$ respiratory syncytial virus; FluA $=$ influenza $A ; * * * I C U=$ Intensive Care Unit; $* * * * \mathrm{OTI}=$ orotracheal intubation; ${ }^{\dagger} p$ value of ANOVA test; ${ }^{\ddagger}$ Kruskal-Wallis test (variance not homogeneous).

\section{Figure 1}

Length of hospitalization (days) of children under 2 years old according to the results of rapid tests RSV, Influenza A and both, PUCCampinas Hospital, 2013 to 2018.

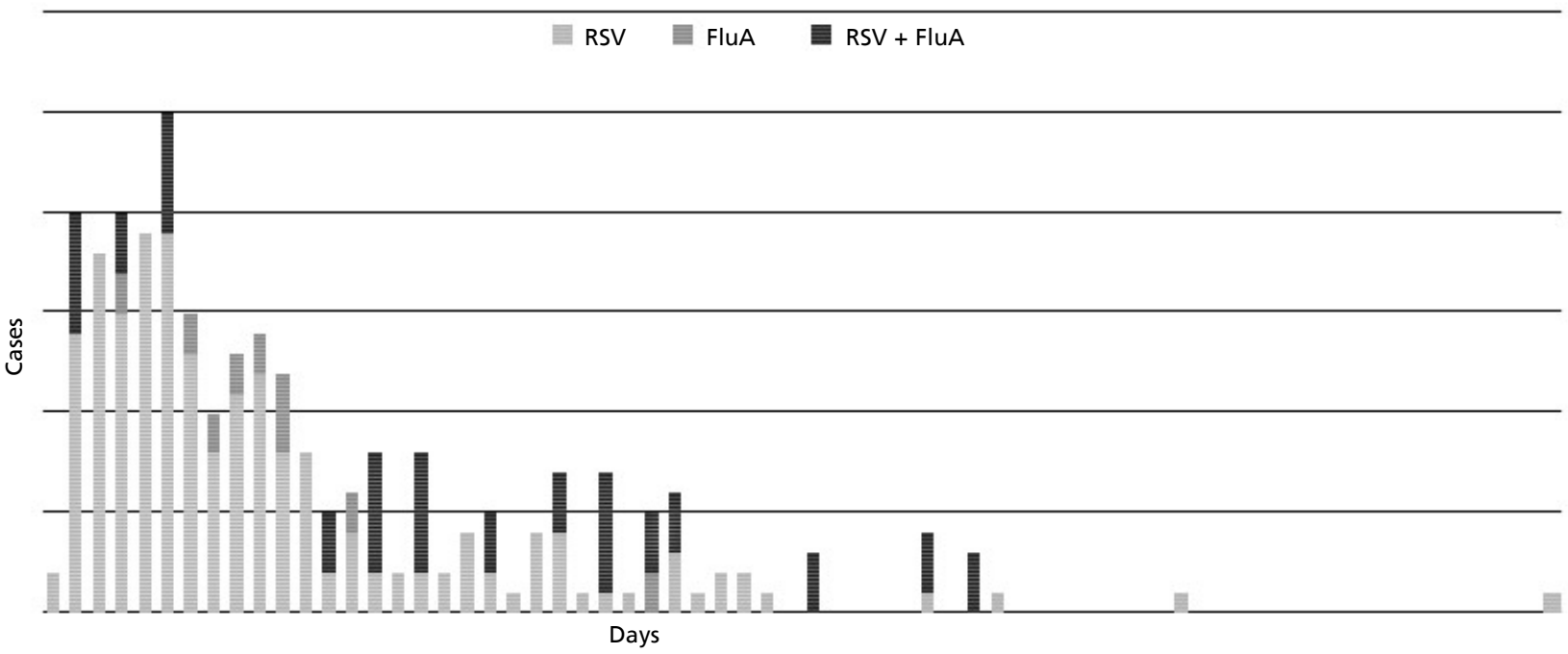

RSV= respiratory syncytial virus; FluA= influenza $A$. 
Table 2

Factors associated with orotracheal intubation (OTI) in children <2 years hospitalized for respiratory viral infection, obtained in a univariate and multiple logistic regression model, PUC-Campinas Hospital, Brazil 2013-2018.

\begin{tabular}{lccccc}
\hline Variable & \multicolumn{2}{c}{ OTI } & & \multirow{2}{*}{ OR crude (CI95\%) } & OR adjustaded (IC95\%) \\
\cline { 2 - 3 } & $\mathrm{n}$ & $\%$ & & $0.94(0.88-0.99)$ & $0.89(0.82-0.98)$ \\
Age in months & & & & & - \\
RT+ * Only RSV & 64 & 34.7 & $0.12(0.42-0.32)$ & - \\
RT+ Only Influenza A & 6 & 66.7 & $3.05(0.74-12.5)$ & $14.3(3.0-68.2)$ \\
RT+ FluA+RSV & 17 & 89.0 & $15.0(3.4-66.9)$ & $2.7(1.02-7.11)$ \\
Comorbidity** & 17 & 58.6 & $1.9(0.9-4.5)$ & - \\
Prematurity (<37 weeks) & 16 & 55.2 & $1.3(0.5-2.9)$ & $4.78(1.83-12.55)$ \\
Associated bacterial pneumonia & 25 & 75.8 & $5.9(2.5-13.8)$ & \\
\hline
\end{tabular}

*RT= Rapid Test; **Comorbidity: congenital heart disease, Down syndrome, other GIT congenital malformations, renal failure, bronchopulmonary dysplasia; $R S V=$ respiratory syncytial virus; FluA= influenza $A$.

Figure 2

Temporal distribution of hospitalization of children $<2$ years old with viral respiratory infection, PUC-Campinas Hospital, 2013 to 2018.

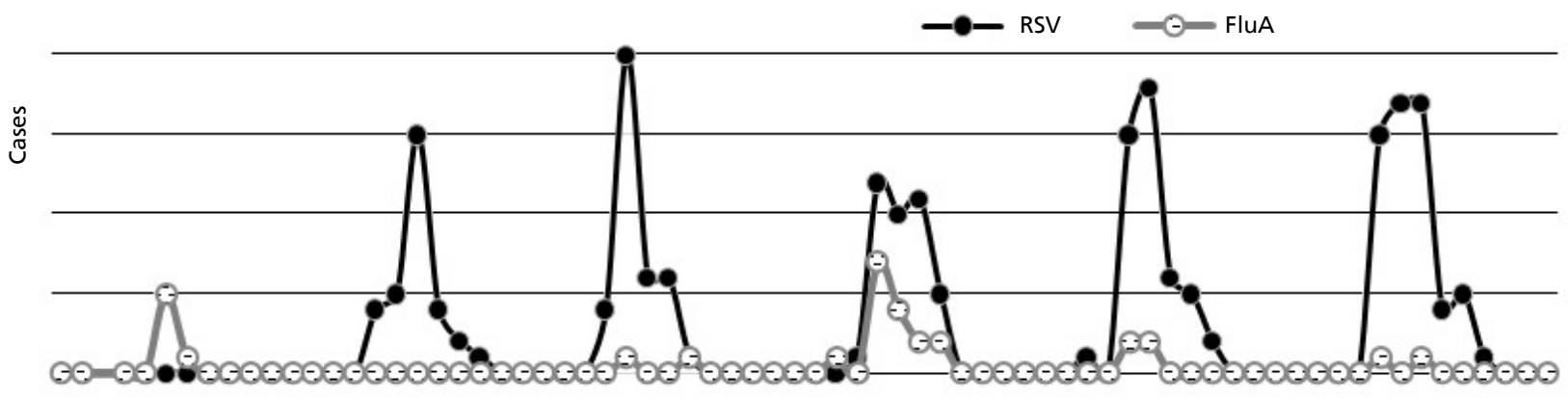

RSV= respiratory syncytial virus; FluA $=$ influenza $A$

are heterogeneous, analyzing different viral combinations, age groups, and outcomes.3,12,15,18-23 Although still controversial, our results corroborate findings from several studies that correlate viral codetection with higher risk of hospitalization.20,21

Diagnostic methods today are extremely sensitive, particularly molecular RT-PCR viral tests with approximately $99 \%$ sensitivity. However, this technology is not accessible in primary care and general hospital settings, and in most cases does not allow real-time diagnosis, because technical expertise is required. 8,9 Conversely, rapid immunochromatographic antigen detection tests, despite lower sensitivity (Flu, 77\%-80\%; RSV, 50\%-94\%), have high specificity $>95 \%), 8,22-25$ and can be an important tool for point-of-care diagnosis. In addition, there is evidence of increased sensitivity of testing in young children due to high viral excretion. 25

Rapid immunochromatographic antigen detection tests allow immediate decision making regarding the introduction of antiviral treatments, improving the effectiveness of infection prevention and control measures, and reducing hospital admission rates. 26 A randomized trial performed in pediatric patients showed that RIDTs result in fewer antibiotic prescriptions and fewer unnecessary investigations, such blood cultures, and Xrays. 27

With the increase in the use of multiplex RTPCR, high co-detection rates of different viruses are identified, ranging from $18 \%$ to $40 \% .11,28$ 
Nevertheless, there is evidence that the seasonality of a virus can interfere competitively with the incidence of other viruses. 27 In our study, we detected ( $4.1 \%$ influenza and $8.6 \%$ influenza plus RSV), which can be considered low, given the active circulation of these viruses in the fall and winter seasons 21,28 (Figure 1). In another study, detection rates for both viruses (RSV and influenza) in hospitalized children were $\geq 8.6 \%$ among children $<1$ year of age. 20

This study has some limitations, such as the retrospective design, the use of rapid diagnostic tests with a lower sensitivity in contrast to RT-PCR, and inclusion of tests only for RSV and influenza, despite other relevant virus as metapneumovirus, adenovirus, and rhinovirus. Moreover, the study of cases from a specific clinical referral service does not allow for analysis of risk factors related to all cases of viral respiratory infection in the community. On the other hand, these tests are potentially accessible in the routine employed in most emergency pediatric care services.

\section{References}

1. Alvarez AE, Marson FA, Bertuzzo CS, Arns CW, Ribeiro JD. Epidemiological and genetic characteristics associated with the severity of acute viral bronchiolitis by respiratory syncytial virus. J Pediatr. 2013; 89: 531-43.

2. Shi T, McAllister DA, O’Brien KL, Simoes EAF, Madhi SA, Gessner BD, Bassels E, Pollak F, Sozinho A, Aguayol C, Alassani I, Asad A, Martim A, Hally A, Awasthi S, Awori JO, Azziz-Baumgartner E, Bagget HC, Baillie VL, Balmaceda A, et al. Global, regional, and national disease burden estimates of acute lower respiratory infections due to respiratory syncytial virus in young children in 2015: a systematic review and modelling study. Lancet. 2017; 390: 946-58.

3. Celik K, Olukman O, Demirol H, Terek D, Gulfidan G, Devrim I, Gulcu P, Arslanoglu S, Calkavur, S. Prevalence of respiratory pathogens during two consecutive respiratory syncytial virus seasons at a tertiary medical care center. Arch Argent Ped.2019; 117 (4): e356-e362.

4. Taylor S, Lopez, P, Weckx L, Borja-Tabora, C, UlloaGutierrez R, Lazcano-Ponce E, Safadi MAP. Respiratory viruses and influenza-like illness: epidemiology and outcomes in children aged 6 months to 10 years in a multicountry population sample. J Infec. 2017; 74: 29-41.

5. Freitas AR, Francisco P, Bergamo MS, Donalisio MR. Mortality associated with influenza in tropics, state of São Paulo, Brazil, from 2002 to 2011: the pre-pandemic, pandemic, and post-pandemic periods. Influenza Res Treat. $2013 ; 696274$.

6. CDC (Centers for Disease Control and Prevention). Yellow Book. Respiratory Infections. [Accessed September 2019]. Available at: https://wwwnc.cdc.gov/travel/yellow-
In conclusion, in pediatric emergence care services, the use of point of care tests can support the early identification of patients at risk for clinical complications. Our study detected high risk of OTI, ICU, and longer hospital stay among children under 2 years of age with co-detection of FluA + RSV.

\section{Author's contribution}

Mendes ET: Laboratory and clinical data collection, conception, article writing, literature review. Paranhos HL: Data collection, conception, article writing. Santos ICM and Souza LB: Data collection, literature review. Aquino JLB, Merhi VAL and Lima MPJS: Data interpretation and manuscript review. Silva RV: Laboratory and clinical data collection, discussion and review of results. All authors approved the final version of the article. book/2020/posttravel-evaluation/respiratory-infections.

7. Debiaggi M, CanducciF, Ceresola ER, Clementi M. The role of infections and coinfections with newly identified and emerging respiratory viruses in children. Virol J. 2012; 9: 247.

8. Bruning $A H$, van Dijk $\mathrm{K}$, van Eijk HW, Koen G, van Woensel JB, Kruisinga FH, PajkrtD, Wolthers KC. Evaluation of a rapid antigen detection point-of-care test for respiratory syncytial virus and influenza in a pediatric hospitalized population in the Netherlands. Diagn Microbiol Infect Dis. 2014; 80: 292-3

9. Ko F, Drews SJ. The impact of commercial rapid respiratory virus diagnostic tests on patient outcomes and health system utilization. Expert Rev Mol Diagn. 2017; 17:91731.

10. Topoulos S, Giesa C, Gatermann S, Fussen R, Lemmen S, Ewig S. Analysis of acute respiratory infections due to influenza virus A, B and RSV during an influenza epidemic 2018. Infection. 2019; 47: 425-33.

11. Martin ET, Kuypers J, Wald A, Englund JA. Multiple versus single virus respiratory infections: viral load and clinical disease severity in hospitalized children. Influenza Other Respir Viruses. 2012; 6: 71-7.

12. De Paulis M, Gilio AE, Ferraro AA, Ferronato AE, do Sacramento PR, Botosso VF, Oliveira DBL, Marinheiro JC, Hársi CM, Durigon EL, Vieira SE. Severity of viral coinfection in hospitalized infants with respiratory syncytial vírus infection. J Pediatr. 2011; 87: 307-13.

13. Asner SA, Science ME, Tran D, Smieja M, Merglen A, Mertz D. Clinical Disease Severity of Respiratory Viral Co- 
Infection versus Single Viral Infection: A Systematic Review and Meta-Analysis. PLoS ONE. 2014; 9 (6): e99392.

14. Amini R, Gilca R, Boucher FD, Charest H, De Serres G. Respiratory syncytial virus contributes to more severe respiratory morbidity than influenza in children $<2$ years during seasonal influenza peaks. Infection. 2019; 23:1-7.

15. Rodríguez DA, Rodríguez-Martínez CE, Cárdenas AC, Quilaguy IE, Mayorga LY, Falla LM, Nino G. Predictors of severity and mortality in children hospitalized with respiratory syncytial virus infection in a tropical region. Pediatr Pulmon. 2014; 49 (3): 269-76.

16. Klein EY, Monteforte B, Gupta A, Jiang W, May L, Hsieh $\mathrm{YH}$, Dugas A. The frequency of influenza and bacterial coinfection: a systematic review and meta-analysis. Influenza Other RespirViruses. 2016; 10: 394-403.

17. Morens DM, Taubenburger JK, Fauci AS. Predominant role of bacterial pneumonia as a cause of death in pandemic influenza: implications for pandemic influenza preparedness. J Infect Dis. 2008; 198: 962-70.

18. Sly PD, Jones CM. Viral co-detection in infants hospitalized with respiratory disease: is it important to detect? J Pediatr. 2011; 87: 277-80.

19. Richard N, Komurian-Pradel F, Javouhey E, Perret M, Rajoharison A, Bagnaud A, Billaud G, Vernet G, Bruno L Floret $\mathrm{D}$, Paranhos-Baccalà $\mathrm{G}$. The impact of dual viral infection in infants admitted to a pediatric intensive care unit associated with severe bronchiolitis. Pediatr Infect Dis J. 2008; 27: 213-7.

20. Lafond KE, Nair H, Rasooly MH, Valente F, Booy R, Rahman M, Kitsutani P, Yu H, Guzman G, Coulibaly D, Armero J, Jima D, Howie SRC, Global Respiratory Hospitalizations - Influenza Proportion Positive (GRIPP) Working Group . Global Role and Burden of Influenza in Pediatric Respiratory Hospitalizations, 1982-2012: A Systematic Analysis. PLoS Med. 2016; 13: e1001977.

21. Meskill SD, Revell PA, Chandramohan L, Cruz AT. Prevalence of coinfection between respiratory syncytial virus and influenza in children. Am J Emerg Med. 2017; 35 495-8
22. Walzl G, Tafuro S, Moss P, Openshaw PJ, HussellT. Influenza virus lung infection protects from respiratory syncytial virus induced immunopathology. J Exp Med. 2000; 192: 1317-26

23. Semple MG, Cowell A, Dove W, Greensill J, McNamara PS, Halfhide C, Shears P, Smyth RL, Hart AC. Dual infection of infants by human metapneumovirus and human respiratory syncytial virus is strongly associated with severe bronchiolitis. J Infect Dis. 2005; 191: 382-6.

24. Principi N, Esposito S. Antigen-Based Assays for the Identification of Influenza Virus and Respiratory Syncytial Virus: Why and How to Use Them in Pediatric Practice. Clin Lab Med. 2009; 29: 649-60

25. Piché-Renaud PP, Turcot J, Chartrand C, Gravel J, Labrecque M, Vallières É, Renaud C. Evaluation of a fluorescent immunoassay rapid test (Sofia ${ }^{\mathrm{TM}}$ ) for detection of influenza $\mathrm{A}+\mathrm{B}$ and RSV in a tertiary pediatric setting. Diagn Microbiol Infect Dis. 2016; 84: 304-8.

26. van Asten L, Bijkerk P, Fanoy E, van Ginkel A, Suijkerbuijk A, van der Hoek W, Meijer A, Vennema H. Early occurrence of influenza A epidemics coincided with changes in occurrence of other respiratory virus infections. Influenza Other RespirViruses. 2016; 10: 14-26.

27. Canducci F, Debiaggi M, Sampaolo M, Marinozzi MC, Berrè S, Terulla C, Gargantini G, Cambieri P, Romero E, Clementi M. Two-year prospective study of single infections and co-infections by respiratory syncytial virus and viruses identified recently in infants with acute respiratory disease. J Med Virol. 2008; 80: 716-23.

28. Cebey-López M, Herberg J, Pardo-Seco J, Gómez-Carballa A, Martinón-Torres N, Salas A, Martinón-Sánchez JM, Gormley S, Sumner E, Fink C, Martinón-Torres F, GENDRES network Viral Co-Infections in Pediatric Patients Hospitalized with Lower Tract Acute Respiratory Infections. PLoS ONE. 2015; 10: e0136526.

Received on September 22, 2020

Final version presented on March 2, 2021

Approved on March 31, 2021 